\title{
Comparación de los Niveles de Hormonas Tiroideas por Sexo en CaninosAdultos
}

\author{
Comparison of Thyroid Hormone Levels by Sex in Adult Dogs \\ José Henry Osorio ${ }^{1,2}$, Yirly Johanna Suárez ${ }^{1}$
}

\section{REsUMen}

\begin{abstract}
El objetivo del estudio fue establecer valores de referencia para las concentraciones séricas de la hormona estimulante de la tiroides (TSH) y tetrayodotironina libre $\left(\mathrm{T}_{4} \mathrm{~L}\right)$ en caninos adultos de la zona de Caldas, Colombia y las posibles diferencias atribuidas al sexo. Las muestras de sangre se recolectaron de 78 caninos enteros (40 hembras y 38 machos mayores de un año), en ayunas, y se determinaron los niveles de TSH y $\mathrm{T}_{4} \mathrm{~L}$ mediante inmunoensayo enzimático con kits comerciales. Los valores de TSH para las hembras fueron de $0.53 \pm 0.96 \mu \mathrm{UI} / \mathrm{ml}$ (rango de 0.02 a 4.54 ) y para los machos de $0.36 \pm$ $0.27 \mu \mathrm{UI} / \mathrm{ml}(0.02$ a 0.91$)$. Los valores de $\mathrm{T}_{4} \mathrm{~L}$ para las hembras fueron de $1.08 \pm 0.40 \mathrm{ng} / \mathrm{dl}$ (0.52 a 2.09) y para los machos de $1.07 \pm 0.39 \mathrm{ng} / \mathrm{dl}(0.5$ a 2.18$)$. No hubo diferencia estadística entre sexos para TSH y $\mathrm{T}_{4} \mathrm{~L}$.
\end{abstract}

Palabras clave: metabolismo, canino, $\mathrm{T}_{4}$ libre, $\mathrm{TSH}$

\section{Abstract}

The aim of this study was to establish reference values for serum levels of thyroid stimulating hormone (TSH) and free tetraiodothyronine $\left(\mathrm{T}_{4} \mathrm{~L}\right)$ in adult dogs in the area of Caldas, Colombia, and possible differences due to sex. Blood samples were collected from 78 intact dogs after fasting (40 females and 38 males older than one year of age). The serum concentration of TSH and $\mathrm{T}_{4} \mathrm{~L}$ levels were determined by enzymatic immunoassay using commercial kits. TSH values for females were $0.53 \pm 0.96 \mu \mathrm{UI} / \mathrm{ml}$ (range: $0.02-4.54$ ) and for males were $0.36 \pm 0.27 \mu \mathrm{UI} / \mathrm{ml}(0.02-0.91)$. $\mathrm{T}_{4} \mathrm{~L}$ values for females were $1.08 \pm 0.40$ $\mathrm{ng} / \mathrm{dl}(0.52-2.09)$, and for males was $1.07 \pm 0.39 \mathrm{ng} / \mathrm{dl}(0.5-2.18)$. None statistical difference was found between sexes for both TSH and T4L.

Key words: metabolism, canine, free $\mathrm{T}_{4}, \mathrm{TSH}$

\footnotetext{
${ }^{1}$ Laboratorio de Bioquímica Clínica y Patología Molecular, Departamento de Ciencias Básicas de la Salud, Universidad de Caldas, Manizales, Colombia

${ }^{2}$ E-mail: jose.osorio_o@ucaldas.edu.co
}

Recibido: 24 de febrero de 2015

Aceptado para publicación: 5 de setiembre de 2015 


\section{INTRODUCCIÓN}

En los caninos, al igual que todos los mamíferos, la glándula tiroides (GT) está implicada en la formación de hormonas tiroideas (3,5,3',5'-tetrayodotironina [ $\mathrm{T}_{4}$ ] y la $3,5,3^{\prime}$ 'triyodotironina $\left[\mathrm{T}_{3}\right]$ ) bajo el control de la adenohipófisis, mediante la secreción de tirotrofina u hormona estimulante de la tiroides (TSH) (Swenson y Reece, 1999; Feldman y Nelson, 2000; Cunningham, 2003; RamírezBenavides y Osorio, 2009; Osorio y LópezSalazar, 2011).

$\mathrm{T}_{3}$ y $\mathrm{T}_{4}$ son hormonas metabólicamente activas, donde el $99 \%$ circula unido a proteínas plasmáticas (globulinas), quedando menos del $1 \%$ libre y, por lo tanto, disponible para unirse a los receptores celulares (Marca et al., 1996; Gobello y Goya, 1998). La GT también produce una hormona inactiva: 3,3 , 5 triyodotironina $\left(\mathrm{rT}_{3} \mathrm{o} \mathrm{T}_{3}\right.$ reverse), la cual no tiene actividad biológica y parece producirse en periodos de enfermedad, inanición o estados de catabolismo endógeno excesivo (Marca et al., 1996; Feldman y Nelson, 2000; Yen, 2001).

Las hormonas tiroideas tienen un papel fundamental en la diferenciación celular, el crecimiento del organismo, la regulación del metabolismo y la homeostasis de los tejidos. Además, en el desarrollo fetal, especialmente en los sistemas neural y esquelético, y estimula la calorigénesis (Feldman y Nelson, 2000; Yen, 2001). En los estados de ansiedad y excitación se produce una disminución en la secreción de TSH, aumentando el metabolismo y, por lo tanto, el calor. Por el contrario, en condiciones de frío se eleva la producción y secreción de TRH y TSH debido al estímulo de los centros hipotalámicos del control de la temperatura (Osorio y LópezSalazar, 2011).

En el canino, las anormalidades estructurales o funcionales de la GT pueden llevar a un déficit en la producción de hormona tiroidea. Por esta razón es importante la determinación de los niveles de $\mathrm{T}_{4}$ Libre $\left(\mathrm{T}_{4} \mathrm{~L}\right)$, ya que mide la cantidad de hormona tiroidea no unida a las proteínas séricas transportadoras; es decir, la cantidad de $\mathrm{T}_{4}$ biológicamente activa (Swenson y Reece, 1999; Ferguson, 2007).

Actualmente, la interpretación correcta de las pruebas de función de la tiroides es el desafío más discutido para los diagnósticos de endocrinología clínica en animales de compañía, teniendo en cuenta que hay factores que afectan las concentraciones basales de las hormonas tiroideas como la edad, raza, temperatura ambiental, estado nutricional y salud, al igual que existe un rango muy amplio de valores 'normales'. Además, es importante enfatizar que las mediciones de T4 total no indican la concentración de hormona libre, que es el elemento de importancia para las células blanco (Swenson y Reece, 1999). Por esta razón, el objetivo del presente estudio fue determinar los niveles de TSH y $\mathrm{T}_{4}$ libre en caninos adultos con el fin de establecer valores de referencia según sexo.

\section{Materiales y Métodos}

Se tomaron, en estado de ayuno, muestras de sangre a 78 caninos enteros criollos y mestizos, mayores de un año, en el departamento de Caldas, Colombia (40 hembras y 38 machos). Los animales estaban clínicamente sanos, y las hembras no estaban gestando ni en etapa de lactancia.

Las muestras se tomaron en horas de la mañana, con vacutainer sin anticoagulante, mediante venopunción en la yugular. Las muestras fueron refrigeradas para su transporte al laboratorio, donde fueron centrifugadas a $3500 \mathrm{rpm}$ (Thermo IEC CL31 Multispeed) durante $15 \mathrm{~min}$. El suero extraído fue conservado a $-30{ }^{\circ} \mathrm{C}$ hasta su análisis. 
En la determinación de $\mathrm{T}_{4}$ libre se utilizó la prueba de inmunoensayo enzimático competitivo (Free Thyroxine [Ft4] AccuBind ELISA Kit, Monobind Inc., EEUU), siguiendo las indicaciones del fabricante. La concentración de los analitos en la curva estándar cubría el rango de 0 a $7.40 \mathrm{ng} / \mathrm{dL}$ (Monobind Inc, 2015). Los sueros fueron colocados en contacto con una fase sólida que contenía anticuerpos contra T4, al cual se le agregó el conjugado compuesto por $\mathrm{T}_{4}$ libre unido a peroxidasa de rábano (HRP), formando una reacción de competición por los sitios de unión. La lectura se hizo en un equipo lector de micro ELISA (TitertekMultiscan ${ }^{\mathrm{TM}}$ ) a una absorbancia de $450 \mathrm{~nm}$.

Para la determinación de los niveles de TSH se utilizó una prueba de inmunoensayo enzimático colorimétrico tipo sandwich, utilizada para la cuantificación del TSH de origen humano (Thyrotropin [TSH] AccuBind ELISA Kit, Monobind Inc., EEUU), siguiendo las indicaciones del fabricante. La concentración de los analitos en la curva estándar cubría el rango de 0 a $40 \mu \mathrm{UI} / \mathrm{ml}$ (Monobind Inc, 2015). Los pozos recubiertos con estreptavidina interactúan con los anticuerpos monocolonales biotinilados anti-TSH y los sueros de las muestras formando una reacción antígeno-anticuerpo en forma de un complejo sándwich soluble. La lectura se hizo en un fotómetro lector de micro ELISA (TitertekMultiscan ${ }^{\mathrm{TM}}$ ) a una longitud de onda de $450 \mathrm{~nm}$.

Los kits fueron validados para su uso en muestras de caninos en el laboratorio del presente estudio, utilizando sueros y plasma sanguíneo, obteniendo buenas curvas de paralelismo. En la validación se trabajó con 50 muestras por triplicado en mediciones intraensayo e inter-ensayo, obteniéndose un coeficiente de variación de 8 y $9 \%$, respectivamente.

Se determinó el promedio, desviación estándar y valores mínimos y máximos para las concentraciones de $\mathrm{TSH}$ y $\mathrm{T}_{4}$ libre por sexo. El análisis estadístico se hizo mediante un análisis de varianza usando el programa Statgraphics Plus 5.1.

\section{Resultados y Discusión}

El Cuadro 1 muestra los valores obtenidos para los niveles de TSH y $\mathrm{T}_{4}$ libre en caninos adultos. No hubo diferencia estadística con una confiabilidad del $95 \%$ al evaluar los niveles de TSH $(\mathrm{p}=0.08)$ y $\mathrm{T}_{4}$ libre $(\mathrm{p}=0.87)$ entre machos y hembras.

El perfil tiroideo encontrado en el presente estudio fue simular a los valores de referencia para $\mathrm{T}_{4} \mathrm{~L}$ reportado por otros autores (Ramsey et al., 1997; Melián-Limiñana et al., 1999; Matamoros et al., 2002; Ramírez-Benavides y Osorio, 2009). Asimismo, la ausencia de diferencia estadística para los valores de TSH como de $\mathrm{T}_{4} \mathrm{~L}$ según sexo ha sido ampliamente reportada (MeliánLimiñana, 1999; Feldman y Nelson, 2000; Ramírez-Benavides y Osorio, 2009; Pessina et al., 2010).

Estudios sobre concentraciones séricas de $\mathrm{T}_{4} \mathrm{y} \mathrm{T}_{3}$ en perros han demostrado que los valores de $\mathrm{T}_{3}$ no se afectan con la edad pero la $\mathrm{T}_{4}$ es inversamente proporcional a la edad, encontrándose aumentada en canes menores de 6 semanas y que los niveles disminuyen a medida de que el perro envejece. En relación al tamaño corporal, la concentración sérica de $\mathrm{T}_{4}$ es mayor en perros pequeños que en medianos y grandes, en tanto que la concentración sérica media de $\mathrm{T}_{3}$ es mayor en perros medianos (Feldman y Nelson, 2000; Ramírez-Benavides y Osorio, 2009).

El establecimiento de valores de referencia para TSH y $\mathrm{T}_{4} \mathrm{~L}$ es de importancia debido a que existen enfermedades de presentación común en caninos como, por ejemplo, trastornos dermatológicos, sistémicos, diabetes mellitus, hiperadrenocortisismo, hiperlipidemias asociadas principalmente a hipotiroidismo, hipertiroidismo y neoplasias 
Cuadro 1. Valores de TSH y $\mathrm{T}_{4}$ libre $\left(\mathrm{T}_{4} \mathrm{~L}\right)$ en caninos mayores de un año de edad

\begin{tabular}{lcccccc}
\hline & \multicolumn{2}{c}{ Hembras } & \multicolumn{2}{c}{ Machos } & \multicolumn{2}{c}{$\mathrm{p}$} \\
\hline Parámetro & $\begin{array}{c}\mathrm{TSH} \\
(\mu \mathrm{UI} / \mathrm{ml})\end{array}$ & $\begin{array}{c}\mathrm{T}_{4} \mathrm{~L} \\
(\mathrm{ng} / \mathrm{dl})\end{array}$ & $\begin{array}{c}\mathrm{TSH} \\
(\mu \mathrm{UI} / \mathrm{ml})\end{array}$ & $\begin{array}{c}\mathrm{T}_{4} \mathrm{~L} \\
(\mathrm{ng} / \mathrm{dl})\end{array}$ & $\mathrm{TSH}$ & $\mathrm{T}_{4} \mathrm{~L}$ \\
\hline Promedio & 0.53 & 1.08 & 0.36 & 1.07 & 0.08 & 0.87 \\
Desviación estándar & 0.96 & 0.40 & 0.27 & 0.39 & & \\
Mínimo & 0.02 & 0.52 & 0.02 & 0.5 & & \\
Máximo & 4.54 & 2.09 & 0.91 & 2.18 & & \\
\hline
\end{tabular}

tiroideas que pueden tener un efecto significativo sobre las concentraciones séricas basales de hormonas tiroideas y sobre la capacidad de respuesta de la tiroides a la TSH y a la TRH.

El hipotiroidismo se puede dividir en tres categorías basado en el sitio de la enfermedad: el tipo primario se da en la glándula tiroides y es la forma más común $(>95 \%$ de los casos), y se debe usualmente a atrofia idiopática de la glándula o tiroiditis linfocítica mediada por el sistema inmune; los tipos secundario y terciario se dan en la adenohipófisis e hipotálamo y son formas de rara presentación (Matamoros et al., 2002).

El mejor parámetro para estimar el eje de la GT es la TSH, ya que es el marcador más sensible para evaluar su funcionalidad, donde niveles elevados de TSH son compatibles con hipotiroidismo primario (75\% de los casos), independientemente de los valores de $\mathrm{T}_{4}$, pero si la TSH está baja, indica que es de carácter secundario o terciario (Matamoros et al., 2002; Castillo, 2011). El 25\% de los casos de hipotiroidismo canino presentan niveles de TSH y $\mathrm{T}_{4} \mathrm{~L}$ normales, pero cerca de los valores límite (superior e inferior), y si hay una firme sospecha de la enfermedad (imagen alterada de glándula tiroidea, presencia de por lo menos un signo clínico compatible con hipotiroidismo), la confirmación diagnóstica de la enfermedad se obtiene mediante la prueba de estimulación con TRH (Castillo, 2011).

\section{Agradecimientos}

Los autores agradecen a Jorge Enrique Pérez Cárdenas del Departamento de Ciencias Básicas de la Salud de la Universidad de Caldas. Manizales, Colombia, por su tiempo y colaboración en la determinación de los perfiles tiroideos.

\section{Literatura Citada}

1. Castillo V. 2011. Hipotiroidismo canino. Revista Vet Focus 21(1): 2-8.

2. Cunningham JG. 2003. Fisiología veterinaria. $3^{a}$ ed. Barcelona, España: Elsevier. $341 \mathrm{p}$.

3. Feldman EC, Nelson RW. 2000. Endocrinología y reproducción en perros y gatos. $2^{\mathrm{a}}$ ed. México DF: McGraw-Hill. $856 \mathrm{p}$.

4. Ferguson DC. 2007. Testing for hypothyroidism in dogs. Vet Clin North Am Small Anim Pract 37: 647-669.

5. Gobello C, Goya R. 1998. Hipotiroidismo canino. CVPBA 7:11-14.

6. Marca MC, Loste A, Sanz MC, Sáez T, Verde MT, Ramos JJ. 1996. Hipotiroidismo canino: revisión y actualización de su diagnostico. Clín Vet Peq Anim 16(2): 111-117.

7. Matamoros R, Gómez C, Andaur M. 2002. Hormonas de utilidad diagnóstica en Medicina Veterinaria. Arch Med Vet 34: 167-182. doi: 10.4067/S0301$732 \times 2002000200003$ 
8. Melián-Limiñana C, Morales-Doreste M, Pérez-Alenza MD. 1999. Concentración de TSH endógena: un nuevo test para el diagnóstico de hipotiroidismo canino. Clín Vet Peq Anim 19(2): 71-75.

9. Melián-Limiñana C. 1999. Diagnóstico de hipotiroidismo canino e hipertiroidismo felino. Vector Plus 13(1): 4-18.

10. Osorio JH, López-Salazar C. 2011. Actualización en el funcionamiento de la glándula tiroides en caninos. Primera parte: funcionamiento normal. Biosalud 10(1): 99-112.

11. Pessina P, Sosa C, Araújo M, Orellana $B$, Brambillasca $S$, Cajarville $C$, Meikle A. 2010. Perfiles metabólicos y endócrinos en perros sanos: influencia de la ingesta y el sexo. Veterinaria 46(177180): 33-38.
12. Ramírez-Benavides GF, Osorio JH. 2009. Niveles séricos de tetrayodotironina libre $\left(\mathrm{T}_{4} \mathrm{~L}\right)$, mediante el método de electroquimioluminiscencia en caninos. Rev Cient FCV-LUZ 19: 238- 241.

13. Ramsey LK, Evans H, Herrtage ME. 1997. Thyroid stimulating hormone and total thyroxine concentrations in euthyroid, sick euthyroid and hypothyroid dogs. J Small Anim Pract 38: 540-545. doi: 10.1111/j.1748-5827.1997.tb03313.x

14. Swenson MJ, Reece WO. 1999. Fisiología de los animales domésticos de Dukes. $2^{\mathrm{a}}$ ed. Noriega:. Uteha. 925 p.

15. Yen PM. 2001. Physiological and molecular basis of thyroid hormone action. Phys Rev 81: 1097-1142. 